\title{
Biotecnologia de embriões: II - Análise qualitativa e quantitativa de embriões obtidos de vacas superovuladas para transferência de embriões*
}

\author{
Biotechnology of embryos: II - Quantitative and qualitative analysis of embryos \\ from superovulated cows used in a embryo transfer program
}

\author{
Ismar Araujo de Moraes ${ }^{1}$, Marcio Ricardo Costa dos Santos², Tania Goes de Pinho², Carlos Fernando Marins \\ Rodrigues $^{3}$ e Cláudio Alvarenga de Oliveira ${ }^{4}$
}

\begin{abstract}
Resumo
Amostras de sangue foram obtidas de 16 vacas (11 Bos taurus taurus e 05 Bos taurus indicus) entre o primeiro dia do tratamento com FSH-P e o dia da coleta dos embriões e analisadas por Radioimunoensaio. Os níveis médios de progesterona circulante (P4) foram analisados no início do tratamento (DIN), no momento imediatamente anterior à aplicação da prostaglandina (DPG), 24 horas após (DAPG), no dia da inseminação artificial (DIA), no terceiro (D3PEST) e quarto (D4PEST) dias após o estro e no dia da coleta dos embriões (DCOL). O grau de associação entre a concentração de P4 durante o programa e a qualidade/quantidade dos embriões obtidos, medido pelo teste de correlação de Spearman (rs), mostrou-se significativo para o DIN $(P<0,01$; $r s=0,59 /$ qualidade), para o D3PEST $(P<0,05$; $r s=0,47$ / qualidade e $\mathrm{P}<0,01 ; \mathrm{rs}=0,66 /$ quantidade), para o D4PEST ( $P<0,01 ; r s=0,70$ /qualidade $\mathrm{P} P<0,01 ; \mathrm{rs}=0,77$ /quantidade) e para o $\mathrm{DCOL}(\mathrm{P}<0,01$; $r \mathrm{~s}=0,69$ /qualidade e $\mathrm{P}<$ 0,$01 ;$ rs $=0,69$ /quantidade) .
\end{abstract}

Palavras chave: progesterona, embriōes, superovulação.

\section{Introdução}

Na literatura consultada observou-se que a utilização de gonadotrofina exógena (PMSG ou FSH-P) na estimulação ovariana para a transferência de embriões em bovinos, pode determinar alterações na função citoplasmática e nuclear dos oócitos comprometendo a qualidade dos embriões (Moor et al. 1985). Uma das causas apontadas para estas alterações é a variação dos padrões normais de estrogênio e progesterona (P4) que podem comprometer tanto a qualidade quanto quantidade e viabilidade dos embriões obtidos (Wilmut et al., 1985).

Vários trabalhos foram conduzidos para analisar a correlação existente entre a concentração de P4, nos diferentes momentos do programa de superovulação e coleta de embriōes, e a qualidade e quantidade dos embriões obtidos. Os resultados, entretanto, demonstraram controvérsias. Ao considerar-se esta relação no início do tratamento de superovulação com gonadotrofina, Sreenan \& Gosling (1977); Saumande (1980) e Bolzoni et al. (1991) não demonstraram qualquer correlação, entretanto, Looney (1986); Yadavetal. (1986); Goto et al. (1987); Elsaesseretal. (1981), Boland et al. (1985) e Datta et al. (1992) observaram existir correlação positiva tanto com a qualidade quanto com a quantidade dos embriōes. Schams et al. (1979), Elsaesser et al. (1981), Greve et al. (1983), e Lindsell et al. (1986), demonstraram que a baixa concentração de P4 neste momento está mais freqüentemente associada com a ausência de embriões.

Após o início do tratamento, Tamboura et al. (1985), Jensen et al. (1982), Britt \& Holt, (1988) observaram que o aumento contínuo da concentração de P4 não influenciava na quantidade dos embriões obtidos, mas encontraram

\footnotetext{
*Trabalho financiado pela FINEP/CNPq.

${ }^{1}$ Med. Vet. Prof. MS. Depto. Fisiologia-UFF. Niterói-RJ. Caixa Postal 100086 - CEP 24230-340

${ }^{2}$ Med. Vet. Prof. Dr. Depto Pat. Clin. Vet-UFF. Pesq.do CNPq - Caixa Postal 100086-Niterói-RJ CEP 24230-340.

${ }^{3}$ Med. Vet. Gertec Embriões. Cx. Postal 548, Atibaia-SP CEP 12940.000

${ }^{4}$ Med. Vet. Prof. Dr. Depto. Reprod. Anim-USP. Av. Prof. Lucio Martins Rodrigues Tv. 4 BL. 25, São Paulo-SP.

${ }^{*} a-L A B-S C H E R I N G-U . S . A$.

*b - CIOSIN - LAB COOPERS

${ }^{*} \mathrm{C}-$ ENCON, U.S.A

*d - CULTILAB, BRASIL

*e - NEW-VACUO-LABNEW IND. e COM., MED-LAB, BRASIL.

${ }^{*}$ - COAT-A-COUNT, DPC - U.S.A.
} 
correlação negativa com a qualidade dos mesmos. Estes autores postularaḿ que o aumento da concentração de P4 foi devido a luteinização prematura dos folículos o que determinou um microambiente hormonal incompatível com a liberação de um oócito completamente maduro e com tendência a degeneração. Entretanto Greve et al. (1983) não encontraram qualquer correlação entre a concentração de P4 no início do tratamento e a qualidade dos embriões.

Nas análises feitas no dia da aplicação da prostaglandina, Yang et al. (1992) observaram correlação positiva entre a concentração de P4 e a qualidade dos embriões. Ao considerar vinte e quatro horas após a aplicação da prostaglandina, Saumande \& Batra (1985), Saumande et al. (1985) e Kweon et al. (1987), verificaram que a concentração de P4 decresceu acentuadamente para níveis inferiores a $0,5 \mathrm{rg} / \mathrm{ml}$ de plasma e permanecia nestes níveis até 0 terceiro dia após o cio. Estes autores concluíram que a baixa concentração de P4 neste momento correlacionava-se positivamente com a quantidade dos embriões obtidos, mas não com a qualidade dos mesmos. Por outro lado, altas concentrações de P4 durante o estro nas fêmeas superovuladas, determinava um efeito negativo sobre a qualidade dos embriões obtidos (Jensen et al., 1982; Greve et al., 1983, Looney, 1986 e Domeki, 1991). Já Tamboura et al. (1985) ao analisarem a concentração deste hormônio no dia da inseminação artificial e no segundo e terceiro dias, verificaram a existência de correlação positiva com o número total de embriões obtidos, mas não com a qualidade dos mesmos.

Determinação dos níveis de P4 no segundo e terceiro dias após o estro subseqüente ao tratamento, por Jensen et al. (1982), Maurer \& Echternkamp (1982) e Maurer \& Echternkamp, (1985), demonstraram que a produção de embriões bovinos transferíveis foi maior quando o aumento da concentração do hormônio ocorreu a partir do terceiro dia após o estro.

Britt \& Holt (1988) observaram ainda, que o rápido aumento da P4 até o segundo e o terceiro dias após o estro induzido, determinava assincronia entre alguns embriōes e o útero, o que diminuía o número de embriões transferíveis. Segundo os autores, esta assincronia provavelmente ocorria quando o último oócito era ovulado muitas horas após o primeiro. Nesta situação, o último embrião estaria fora de sincronia com o útero controlado pela P4 secretada pelos corpos lúteos formados após as primeiras ovulações. Nakajima et al. (1992) ao analisarem o terceiro, quarto e quinto dias após o estro da vaca superovulada, não observaram a existência de qualquer correlação entre a concentração de P4 e a resposta ovariana.

\section{Material e Métodos}

No presente experimento foram utilizadas 16 fêmeas bovinas (11 taurinas e 5 zebuinas), entre 3 e 15 anos de idade, selecionadas para o programa de transferência de embriōes da Gertec Embriões, em Atibaia, Estado de São Paulo.

Na superovulação foram utilizadas 24 a 32mg de FSH$P * a$ por via intra-muscular em doses decrescentes, duas vezes ao dia, durante quatro dias consecutivos. Iniciou-se o programa no décimo dia (D10) após o dia do estro natural (DO). Os animais foram sincronizados com aplicações intramuscular de cloprostenol ${ }^{\text {*b }}$ sendo a primeira, 48 horas após iniciado o tratamento com FSH-P, e a segunda, 12 horas após a primeira.

A inseminação artificial consistiu de duas aplicações, sendo a primeira 48 horas e a segunda 60 horas após a injeção de prostaglandina. A coleta de embriões foi realizada no sétimo dia após a inseminação artificial, utilizando-se sistema fechado de lavagem uterina conforme descrito por Elsden \& Seidel (1982). Os embriões foram recolhidos em Encon Filter ${ }^{\star c}$ e o meio utilizado para lavagem do útero, foi PBS-Dulbecco's modificado*d (Whitinghan, 1971), a $37^{\circ} \mathrm{C}$. Após a coleta os embriões foram identificados como mórulas (MOR), blastocistos (BLAST), degenerados (DEG) e não fecundados (NF) e de acordo com a aparência morfológica, os embriões foram classificados como transferíveis (TRANS) ou não (NTRANS), segundo os critérios de Kusan (1986).

A coleta das amostras de sangue para obtenção de plasma foi realizada através de venopunção jugular com utilização de vacutainer heparinizados*e. As amostras foram obtidas no início do tratamento com a gonadotrofina (DIN), no moḿnento imediatamente anterior à primeira aplicação de prostaglandina (DPG), vinte e quatro horas após a aplicação da primeira dose de prostaglandina (DAPG), no momento da primeira inseminação artificial (DIA), nos terceiro (D3PEST) e quarto (D4PEST) dias após as inseminações e no dia da coleta dos embriões (DCOL), sempre às sete horas.

O plasma obtido por centrifugação, foi imediatamente congelado à temperatura de $-20^{\circ} \mathrm{C}$ sendo assim mantidos até o dia da análise hormonal. A dosagem da progesterona foi feita por radioimunoensaio seguindo-se o protocolo existente nos Kits utilizados ${ }^{* f}$ e os resultados obtidos expressos por $\mathrm{ng} / \mathrm{ml}$ de plasma. Durante o monitoramento hormonal avaliou-se o coeficiente de variação intra e interensaio, para os valores minimos e máximos da concentraçãode progesterona. A análise estatística foi realizada pelo sistema SAEG (1983) para o cálculo das medidas de posição, dispersão e de associação (Teste de correlação de Spearman-rs).

\section{Resultados}

No início do tratamento (DIN) a progesterona endógena atingiu nível médio de $2,42 \pm 1,16 \mathrm{ng} / \mathrm{ml}$. No momento imediatamente anterior à aplicação da prostaglandina (DPG) foi $3,68 \pm 2,64 \mathrm{ng} / \mathrm{ml}$ e 24 horas após (DAPG) observou-se 0,34 $\pm 0,24 \mathrm{ng} / \mathrm{ml}$. Foi observado $0,20 \pm 0,24 \mathrm{ng} / \mathrm{ml}$ no dia da inseminação artificial (DIA), e 1,17 $\pm 1,26 \mathrm{ng} / \mathrm{ml}$ e 3,88 \pm $3,93 \mathrm{ng} / \mathrm{ml}$ para os terceiro (D3PEST) e quarto (D4PEST) dias após o estro, respectivamente. No dia da coleta dos embriões (DCOL) observou-se 15,79 $\pm 14,06 \mathrm{ng} / \mathrm{ml}$.

O número médio de estruturas obtidas por doadora foi 4,30 $\pm 4,23$ embriões transferíveis $(2,60 \pm 4,15$ blastocistos e 1,69 $\pm 2,18$ mórulas) e 3,30 \pm 4,76 embriões não transferíveis (1,52 $\pm 1,95$ degenerados e 1,78 $\pm 3,64$ não fecun- 
dados), perfazendo um total de 7,60 $\pm 6,61$ embriões coletados. Os coeficientes de variação intra e inter-ensaio foram respectivamente $11 \%$ e $25 \%$.

\section{Discussão}

No presente estudo observou-se que a concentração de progesterona no dia do início do tratamento com gonadotrofina está correlacionada com a qualidade dos embriões obtidos ( $r s=0,59 ; P<0,01$ ). Esta correlação já havia sido demonstrada por Looney (1986), Yadav et al. (1986), Goto et al. (1987) e Goto et al. (1988), assim como por Elsaesser et al. (1981), Boland et al. (1985) e Datta et al. (1992) que associaram a baixa qualidade do embrião com concentrações mínimas de P4 no início do tratamento da superovulação. Entretanto, para a quantidade de embriões neste mesmo momento, não houve qualquer associação com a P4 circulante, conforme já demonstrado anteriormente por Looney (1986), Walton \& Stubbings (1986) e Bolzoni et al. (1991). A existência dessa correlação foi, no entanto, verificada no trabalho de Goto et al. (1988).

Os achados do presente estudo no tocante à qualidade dos embriões, diferem dos trabalhos de Sreenan \& Gosling (1977) e de Saumande (1980) sem correlaçõs significativas sobre a resposta ovariana. Também diferem dos achados de Schams et al. (1979), Elsaesser et al. (1981), Greve et al. (1983) e Lindsell et al. (1986) que associaram a ausência de embriōes com uma baixa concentração de P4 no dia do início do tratamento de superovulação. No presente experimento não observou-se existência de coleta sem obtenção de embriões.

A análise estatística para correlação, não demonstrou associação entre a concentração de P4 imediatamente anterior à primeira dose de prostaglandina e a qualidade ou quantidade de embriões obtidos. Tal fato também foi observado por Greve et al. (1983).

Com relação à concentração plasmática de progesterona vinte e quatro horas após a aplicação da primeira dose de prostaglandina não verificou-se a existência de, correlação com a qualidade ou quantidade dos embriões obtidos, diferindo das observações de Jensen et al. (1982), Greve et al. (1984), Saumande \& Batra (1985) e Tambouraet al. (1985), que encontraram a correlação negativa para o número de embriões.

Com relação à concentração plasmática de P4 no dia da inseminação artificial, observou-se neste experimento inexistir correlação entre os níveis atingidos e a qualidade e quantidade de embriões. Na literatura consultada, entretanto, foi reportada a correlação negativa referente à qualidade dos embriões (Jensenet al., 1982; Greveetal., 1983; Looney, 1986 e Domeki, 1991). Saumande et al. (1985), encontraram uma correlação significativa ( $r s=0,33 ; P<0,01$ ) para o número total de embriões obtidos em seus estudos. No presente experimento a inexistência de associação entre os parâmetros considerados, prende-se ao fato de não ter sido observado situação de alta concentraão de P4 neste momento. Entre os animais trabalhados observöu-se níveis inferiores a $0,5 \mathrm{ng} / \mathrm{ml}$ com pequena variação dentro dos valores médios apresentados.
Para os valores médios de progesterona plasmática nos terceiro e quarto dias após o estro, observou-se que no terceiro dia houve correlação significativa com o número de embriões transferíveis ( $r s=0,47 ; P<0,05)$ e com o número total de embriões ( $r s=0,66$; $P<0,01$ ) o que caracterizou a influência desses nívèis sobre a qualidade e quantidade das estruturas obtidas.

Os dados do presente experimento referentes ao terceiro dia após o estro, assemelham-se aos de Jensen et al. (1982) Greve et al. (1983); Saumande et al. (1985); Kweon et al. (1987) que associaram a maior concentração de P4, neste momento, com maior quantidade de embriões obtidos, porém, estes mesmos autores não observaram a existência de correlação significativa no que concerne à qualidade dos embriões. Já no quarto dia após o estro, também observou-se a existência de correlação significativa para o número de embriões transferíveis ( $r s=0,70 ; P<0,01$ ) e para o número total de embriões ( $r s=0,77 ; P<0,01$ ). Estes resultados diferem daqueles apresentados por Nakajima et al. (1992) onde observou-se a inexistência de correlações entre a concentração de P4 e os parâmetros de qualidade e quantidade de embriões.

Com relação aos níveis demonstrados no quarto dia após o estro e às associações verificadas neste experimento, observou-se que os dados apresentados estão de acordo com Saumande et al. (1985) e Kweon et al. (1987) que demonstraram correlação positiva com o total de estruturas coletadas, embora não tenham observado a correlação deste nível com a qualidade dos embriões.

No presente experimento foi observado correlação positiva entre a concentração de P4 no terceiro e quarto dias após o estro e a qualidade dos embriões obtidos. Na literatura consultada, entretanto, não foi observada qualquer citação da referida associação, o que sugere ser esta a primeira caracterização da correlação entre tais parâmetros. Sabe-se que a P4 elevada nos dias subseqüentes ao estro interfere no controle do ístmo da tuba e acarreta prejuízo na qualidade de embriōes recuperados (Hunter, 1985). Os estudos de Wilmut et al. (1985) e Britt \& Holt (1988) ressaltam que tal situação pode determinar assincronia entre alguns embriões, originados de ovulações tardias, com o ambiente uterino apresentando natureza de secreções e estruturas endometriais incompatíveis com o desenvolvimento embrionário. Os achados do presente experimento permitem sugerir que o aumento da concentração de P4 compromete a qualidade dos embriões, porém, somente quando este aumento for verificado no primeiro e no segundo dias após o estro ter ocorrido.

Foi observado que a P4 circulante no dia da coleta de embriões estava associada positivamente com o número de embriões transferíveis ( $r s=0,69$; $P<0,01$ ). Estes dados estão de acordo com os achados de Looney (1986) e Goto et al. (1988). Também verificou-se a existência de correlação positiva com o número total de embriões obtidos ( $r s=0,69$; $P<0,01$ ) já tendo sido demonstrado na literatura nos trabalhos de Looney (1986) e Goto et al. (1988) 
Conclui-se que os fatores hormonais são fontes de variabilidade da resposta ovariana no que se refere aos aspectos quali-quantitativos das estruturas coletadas. Considerando-se que no Brasil ainda pouco se tem estudado sobre tais variações, principalmente em raças zebuinas, torna-se importante a condução de outros estudos para compreender-se melhor as flutuações hormonais que influenciam os aspectos reprodutivos.

\section{Abstract \\ Biotechnology of embryos: II- quantitative and qualitative analysis of embryos from superovulated cows used in a embryo transfer program.}

Blood samples were collected from sixteen cows (11 Bos taurus taurus and 05 Bos taurus indicus) from the beginning of the treatment with FSH-Puntil the day of the embryo collection. The blood plasma were assayed for progesterone (p4), by Radioimmunoassay, at the beginning of the treatment (DIN), immediately before the prostagladin (DPG) and after twentyfour hours (DAPG), on the day of the artificial insemination (DAl), on the third (D3PEST) and the fourth (D4PEST) day after the estrous and on the day of embryo collection (DCOL). a significative correlation between progesterone level and both quality and quantity of embryos was demonstrated at D'N ( $P$ $<0.01$; rs $=0.59$ /quality and $P<0.05$; rs $=0.46$ /quantity), at D3PEST $(P<0.05 ;$ rs $=0.47$ /quality and $P<0.01 ; r s=0.66 /$ quantity), at D4PEST $(P<0.01$; $r$ s $=0.70$ /quality and $P<$ $0.01 ; r s=0.77 /$ quantity) and at DCOL $(P<0.01 ;$ rs $=0.69 /$ quality and $P<0.01 ; r s=0.69$ /quantity)

Key words: progesterone, embryos, superovulation.

\section{Referências}

BOLAND, M.P., FOULKES, J.A., MACDONNEL, H.F. \& SAUER, M.J. Plasma progesterone concentration in superovulated heifers determined by enzymeimmunoassay and radioimmunoassay. Br. Vet.J., v.141, p. 409415, 1985

BOLSONI, P.A., GREGORY, R.M., BRASS, K.E. \& MATTOS, R.C. Concentração sérica de progesterona em vacas superovuladas com PMSG e FSH para transferência embrionária. IX Congresso Brasileiro de Reprodução Animal - Anais, v.2, p. 284, 1991

BRITT, J.H. \& HOLT, L.C. Endocrinological screening of embryo donors and embryo transfer recipients: A review of research with cattle. Theriogenology, v.29, n.1. p. 189-202, 1988

DATTA, T.K., TANEJA, V.K. \& SANVAL, P.C. Hormonal profile in superovulated Hariana cows. Indian Journal of Animal Science $v$. 62, n.8, p.720-722, 1992. Domeki, I. Endocrinology of embryo donor cows. Japanese Journal of Animal Reproductive, v.37, n. 5, p.5764, 1991.

DOMEKI, I. Endocrinology of embryo donor cow. Japanise Journal of Animal Reproduction; v.37, n.5, p. 57-64, 1991

ELSAESSER, F., SACHER, B., HAUPT, P., SCHUTZBAR, W.V. \& SMIDT, D. Relationship between the concentration of progesterone in milk and ovarian response to superovulation treatment in the cow. Zuchthygiene, v. 16, p. 193-200, 1981

ELSDEN, R.P. \& SEIDEL, G.E. Jr. Embryo transfer procedures for cattle Animal Reproduction Laboratory. Colorado State University, 1982.

GOTO, K., NAKANISHI, Y., OHKUTSU, S., OGAWA, K., TASAKY, M., OHTA, H., INOHAE, S., TATEYAMA, S. \& KAWABATA, T. Plasma progesterone profile and embryo quality in superovulated Japonese Black cattle.
Theriogenology, v.27, p.819-826, 1987

GOTO, K., OHKUTSU, S., NAKANISHI, Y., OGAWA, K., TASAKI, M., OHTA, H., INOHAE, S., TATEYAMA, S., KAWABATA, S., ISHII, S., MYIAMOTO, A., FURUSAWA, T., UMEZU, M. \& MASAKI, J. Endocrine profiles and embryo quality in superovulated Japonese Black cattle. Theriogenology, v. 29, p. 615-629, 1988.

GREVE, T., CALLENSEN, H. \& HYTTEL, P. Endocrine profiles and egg quality in the superovulated cow. Nord.Vet. Med., v. 35, n. 11, p. 408-421, 1983.

GREVE, T., CALLENSEN, H. \& HYTTEL, P. Characterization of plasma LH profiles in superovulated dairy cows. Theriogenology, v. 21, p. 237 abst. 1984.

HUNTER, R.H.F. Fertility in cattle:Basic reasons why late insemination must be avoided. Anim. Breed.Abst., v.53, n.2, p. 83-87, 1985

JENSEN, A.M., GREVE, T., MADEJ, A. \& EDQVIST, L.E. Endocrine profiles ad embryo quality in the PMSG-PGF2 $\alpha$ treated cow. Theriogenology, v. 18, p.33-34, 1982.

KWEON, O.K., KANAGAWA, H., TAKAHASHI, Y. \& AOYAGI, Y. Plasma endocrine profiles and total plasma cholesterol levels in superovulated cows. Theriogenology, v. 27, n. 6, p. 841-857, 1987

KUSAN, F.B. Classification of embryos prior to freezing. Short Course of Techniques for Freezing Mammalian Embryos, 1986, Fort Collins. Proceedings. Fort Collins, Animal Reproduction Laboratory ColoradoState University, p. 33-34, 1986.

LINDSELL, C.E.. MURPHY, B.D. \& NAPLETOFT, R.J. Superovulatory and endocrine responses in heifers treated with $\mathrm{FSH}$-pat different stages of the estrous cycle. Theriogenology, v.26, n. 2, p. 209-219, 1986.

LOONEY, R.C. Superovulation in beef females. Proc.Amer. Embryo Transf. Assoc., p. 16-29, 1986.

MAURER, R.R. \& ECHTERNKAMP, S.E. Hormonal assynchrony and embryonic development. Theriogenology, v. 17, n. 1, p. 11-22, 1982

MAURER, R.R. \& ECHTERNKAMP, S.E. Repeat breeder females in beef cattle: influences and causes. J. Anim. Sci., v. 61, n. 3, p. 624-636, 1985.

MOOR, R.M., OSBORN, J.C. \& CROSBY, I.M. Gonadotrophin-induced abnormalities in sheep oocytes after superovulation. J. Reprod. Fertil., v. 74, p. 167-172, 1985.

NAKAJIMA, A., SUZUKI, H., HIRAIZUMI, S., ONODERA, K., SENBOKU, T. \& DOMEKI, I. Preovulatory plasma progesterone and estradiol 17B concentrations and superovulatory response in PMSG-treated donor cows. Animal Science and Technology, v. 63, n. 7, p. 704707, 1992.

SAEG - Sistema para Análises Estatísticas. Divisão de Informática\& Fundação Arthur Bernardes. Universidade Federal de Viçosa, 1983.

SAUMANDE, J. Concentration of luteinizing hormone, oestradiol 17B and progesterone in the plasma of heifers treated to induce superovulation. J. Endocrin., v.84, p. 425-437, 1980

SAUMANDE, J. \& BATRA, S.K. Superovulation in the cow: comparison of oestradiol-17B and progesterone patterns in milk and plasma of cow induced to superovulate; relationships with ovarian responses. J. Endocrinol., v. 107, p. 259-264, 1985

SAUMANDE, J., TAMBOURA, D. \& CHUPIN, D. Changes in milk and plasma concentration of progesterone in cows after treatment to induce superovulation and their relationships with number of ovulation and of embryos collected. Theriogenology, v. 23, n. 5, p. 719-731, 1985

SCHAMS, D., MENZER, Ch., SCHALLENBERGER, E., HOFFMANN, B., PROKOPP, A., HAHN, B. \& HAHN, R. Superovulation in cattle: Hormonal profiles during superovulation with PMSG or Pituitary FSH. Zuchthyngiene, v. 14, p. 11-25, 1979

SREENAN, J.M. \& GOSLING, J.P. The effect of cycle stage and plasma progesterone levels on induction of multiple ovulation in heifers.J. Reprod.Fertil., v. 50, p. 367-369, 1977.

TAMBOURA, D., CHUPIN, D. \& SAUMANDE, J. Superovulation in cows: a relationship between progesterone secretion before ovulation and the quality of embryos. Anim. Reprod. Scie., v. 8, p. 327-334, 1985 
WALTON, J.S. \& STUBBINGS, R.B. Factors affecting the yield of viable embryos by superovulated Holstein-friesian cows. Theriogenology, v.26, n. 2, p. 167-177, 1986.

WHITTINGHAN, D.G. Survival of mouse embryos after freezing and tawing. Nature, n. 233,p.125, 1971.

WILMUT, I., SALES D.I. \& ASHWORTH, C.J. The influence of variation in embryo stage and maternal hormone profiles on embryo survival in farm animals. Theriogenology, v. 23, n. 1, p. 107-117, 1985.
YADAV, M.C., WALTON, J.S. \& LESLIE, K.E. Plasma concentrations of luteinizing hormone and progesterone during superovulation of dairy cows using follicle stimulating hormone or pregnant mare serum gonadotrophin. Theriogenology, v. 26, n. 4, p. 523-540, 1986.

YANG, B.S., OH, S.J., JUNE, J.K., CHO, B.D., IM, K.S. \& LEE, K.S. Endocrine profiles and embryo production in superovulated cows. Research Reports of the Rural Development Administration, v.34, n. 2, p. 1-5, 1992. 